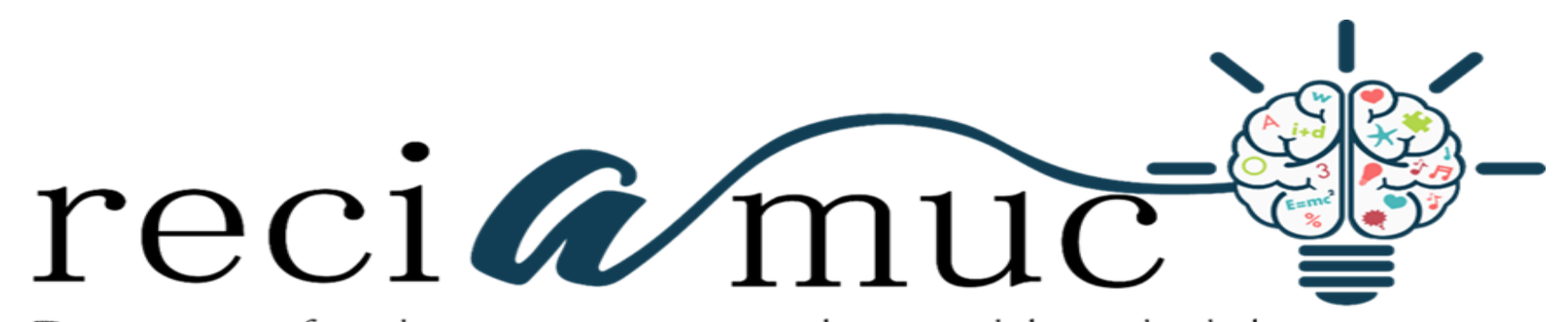

Revista cientifica de investigación actualización del mundo de las ciencias

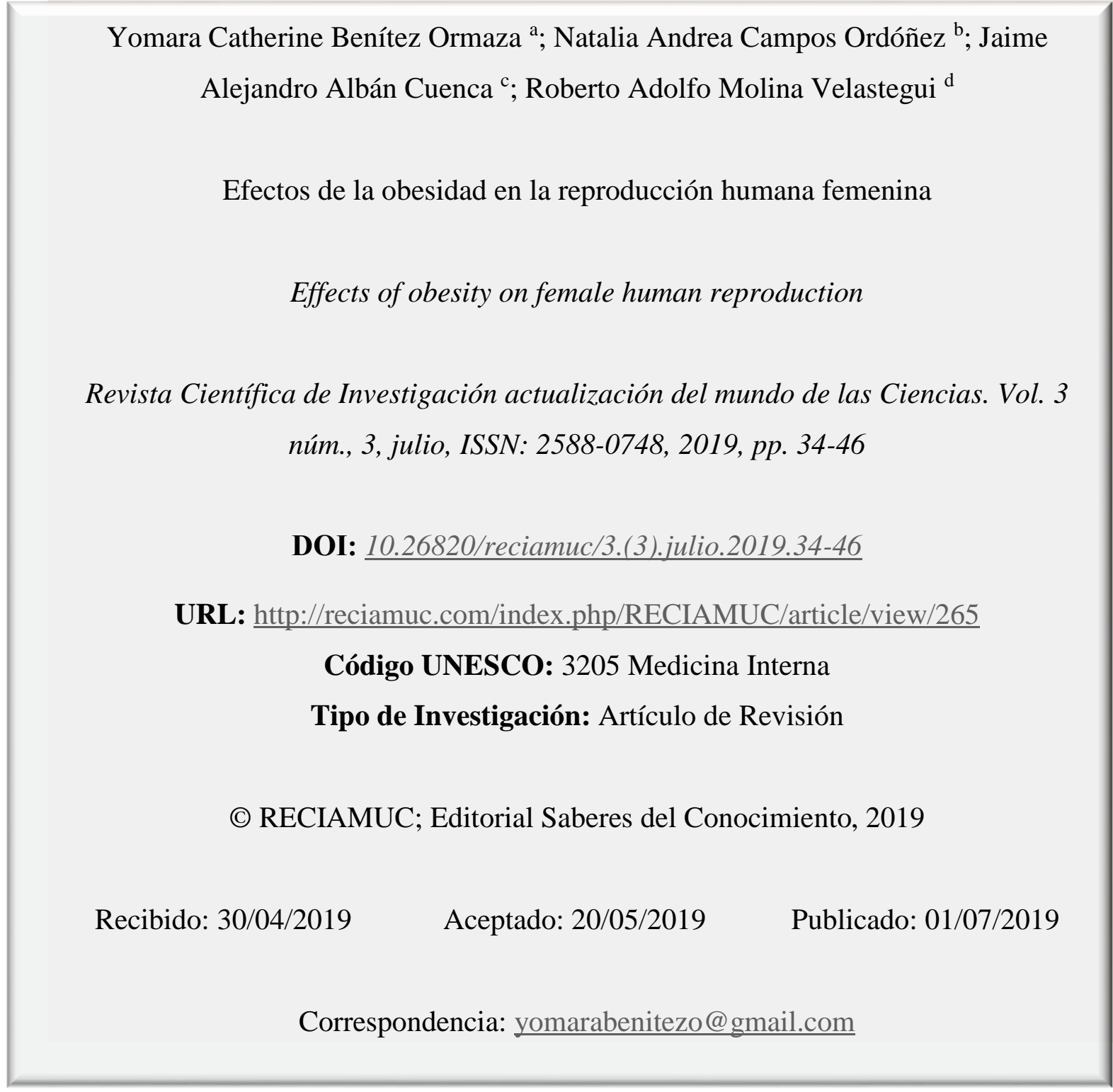

a. Médica; Investigador Independiente; Guayaquil, Ecuador; yomarabenitezo@ gmail.com

b. Médico; Investigador Independiente; Guayaquil, Ecuador; naty14_08@ hotmail.com

c. Médico; Investigador Independiente; Guayaquil, Ecuador; jaimealbancuenca@hotmail.com

d. Doctor en Medicina y Cirugía; Investigador Independiente; Guayaquil, Ecuador; robspidey@ hotmail.com 


\section{Efectos de la obesidad en la reproducción humana femenina}

Vol. 3, núm. 3., (2019)

Yomara Catherine Benítez Ormaza; Natalia Andrea Campos Ordóñez; Jaime Alejandro Albán Cuenca; Roberto Adolfo Molina Velastegui

\section{RESUMEN}

La obesidad tiene un impacto adverso significativo en el resultado reproductivo. Influye no solo en la posibilidad de concepción sino también en la respuesta al tratamiento de fertilidad, y aumenta el riesgo de aborto espontáneo, anomalías congénitas y complicaciones del embarazo, además de los posibles efectos adversos en la salud a largo plazo de la madre y el bebé. Las mujeres deben aspirar a un IMC normal antes de iniciar cualquier forma de tratamiento de fertilidad. El tratamiento debe diferirse hasta que el IMC sea inferior a $35 \mathrm{~kg} / \mathrm{m} 2$, aunque en aquellos con más tiempo (por ejemplo, menos de 37 años; concentración sérica normal de FSH) una reducción de peso a un IMC de menos de $30 \mathrm{~kg} / \mathrm{m} 2$ es preferible. Los médicos deben considerar aplazar el tratamiento a las mujeres fuera de estas pautas. Las mujeres deben ser provistas de asistencia para perder peso, que incluye apoyo psicológico, consejos dietéticos, clases de ejercicios y de adecuados agentes reductores de peso o cirugía bariátrica. Incluso una pérdida de peso moderada de 5 a $10 \%$ del peso corporal puede ser suficiente para restablecer la fertilidad y mejorar los marcadores metabólicos.

Palabras Claves: Obesidad; Fertilidad; Infertilidad; Concepción; Aborto espontáneo; Aborto espontáneo; Complicaciones del embarazo. 


\title{
Efectos de la obesidad en la reproducción humana femenina
}

Vol. 3, núm. 3., (2019)

Yomara Catherine Benítez Ormaza; Natalia Andrea Campos Ordóñez; Jaime Alejandro Albán Cuenca; Roberto Adolfo Molina Velastegui

\begin{abstract}
Obesity has a significant adverse impact on the reproductive outcome. It influences not only the possibility of conception but also the response to fertility treatment, and increases the risk of spontaneous abortion, congenital anomalies and complications of pregnancy, as well as possible adverse effects on the long-term health of the mother and the mother. baby. Women should aim for a normal BMI before starting any form of fertility treatment. The treatment should be deferred until the BMI is less than $35 \mathrm{~kg} / \mathrm{m} 2$, although in those with more time (for example, less than 37 years, normal serum FSH level) a weight reduction at a BMI of less than $30 \mathrm{~kg} / \mathrm{m} 2$ is preferable. Physicians should consider deferring treatment to women outside of these guidelines. Women should be provided with assistance to lose weight, which includes psychological support, dietary advice, exercise classes and adequate weight-reducing agents or bariatric surgery. Even a moderate weight loss of 5 to $10 \%$ of body weight may be enough to restore fertility and improve metabolic markers.
\end{abstract}

Key Words: Obesity; Fertility; Infertility; Conception; Miscarriage; Congenital anomalies; Complications of pregnancy. 


\section{Efectos de la obesidad en la reproducción humana femenina}

Vol. 3, núm. 3., (2019)

Yomara Catherine Benítez Ormaza; Natalia Andrea Campos Ordóñez; Jaime Alejandro Albán Cuenca; Roberto Adolfo Molina Velastegui

\section{Introducción.}

La obesidad es un problema común entre las mujeres en años reproductivos, con un alto porcentaje de mujeres con sobrepeso u obesidad trae consigo un impacto negativo en la concepción espontánea, aborto involuntario, el embarazo y la salud a largo plazo de ambos madre e hijo debido tanto a una mayor tasa de anomalías congénitas y la posibilidad de enfermedad de metabolismo en la vida posterior. La obesidad también tiene un efecto negativo en la fertilidad masculina, pero está más allá del alcance de este trabajo. Las mujeres obesas responden menos a las drogas utilizadas para la estimulación ovárica para el tratamiento de anovulación y concepción asistida, aunque esta no siempre equivale a una reducción en las tasas de embarazo. Además, la obesidad puede afectar la seguridad de los procedimientos, por ejemplo, la capacidad de ver ovarios en ecografía o la provisión segura de anestesia para laparoscopia o recuperación de ovocitos. La obesidad también tiene un gran impacto durante el embarazo y el nacimiento.

Una vez que las mujeres obesas están embarazadas, su riesgo de complicaciones durante el embarazo es significativamente mayor que sus contrapartes delgadas. Según lo analizado por (Catalano, 2010) en este número, muchos de los resultados adversos de la obesidad (incluida la diabetes gestacional y la preeclampsia) se producen como resultado del aumento de la resistencia a la insulina. Incluso las mujeres embarazadas magras son resistentes a la insulina en comparación con sus contrapartes no embarazadas, con resistencia a la insulina mediada aguas abajo del receptor de insulina por defectos en la movilización de SLC2A4 (GLUT4). Es probable que esto sea inducido por factores circulantes, con citoquinas como el factor de necrosis tumoral- $\alpha$ entre los principales candidatos.

Este artículo sintetiza la evidencia sobre la interacción entre la obesidad y la reproducción femenina y presentar orientaciones para potenciar las buenas prácticas clínicas en su gestión. Está dirigido a todos los profesionales de la salud y proveedores de servicios involucrados en la atención a mujeres obesas antes y durante el embarazo. El alcance del artículo incluye los efectos de la obesidad en la fertilidad natural y el resultado del embarazo, el impacto del síndrome de ovario

poliquístico y resistencia a la insulina y la gestión de la anovulación y FIV. También incluye la valoración de los pacientes obesos y medidas para ayudar a las mujeres a perder peso. Los temas 


\section{Efectos de la obesidad en la reproducción humana femenina}

Vol. 3, núm. 3., (2019)

Yomara Catherine Benítez Ormaza; Natalia Andrea Campos Ordóñez; Jaime Alejandro Albán Cuenca; Roberto Adolfo Molina Velastegui

para debate incluyen sí o no debe haber un peso corporal por encima del cual no deben proporcionarse cualquier tratamiento, y si hay evidencia para apoyar tal recomendación basada en la posibilidad de concepción o seguridad durante el tratamiento y el embarazo.

\section{Metodología.}

Para el desarrollo de este proceso investigativo, se plantea como metodología la encaminada hacia una orientación científica particular que se encuentra determinada por la necesidad de indagar en forma precisa y coherente una situación, en tal sentido (Davila, 2015) define la metodología "como aquellos pasos previos que son seleccionados por el investigador para lograr resultados favorables que le ayuden a plantear nuevas ideas".(p.66)

Lo citado por el autor, lleva a entender que el desarrollo de la acción investigativa busca simplemente coordinar acciones enmarcadas en una revisión bibliográfica con el fin de complementar ideas previas relacionadas con los efectos de la obesidad en la reproducción humana femenina a través de una revisión de literatura, para así finalmente elaborar un cuerpo de consideraciones generales que ayuden a ampliar el interés propuesto.

\section{Tipo de Investigación.}

Dentro de toda práctica investigativa, se precisan acciones de carácter metodológico mediante las cuales, se logra conocer y proyectar los eventos posibles que la determinan, así como las características que hacen del acto científico un proceso interactivo ajustado a una realidad posible de ser interpretada. En este sentido, se puede decir, que la presente investigación corresponde al tipo documental, definido por Castro (2016), "se ocupa del estudio de problemas planteados a nivel teórico, la información requerida para abordarlos se encuentra básicamente en materiales impresos, audiovisuales y /o electrónicos”. (p.41).

En consideración a esta definición, la orientación metodológica permitió la oportunidad de cumplir con una serie de actividades inherentes a la revisión y lectura de diversos documentos donde se encontraron ideas explicitas relacionadas con los tópicos encargados de identificar a cada característica insertada en el estudio. Por lo tanto, se realizaron continuas interpretaciones con el 


\section{Efectos de la obesidad en la reproducción humana femenina}

Vol. 3, núm. 3., (2019)

Yomara Catherine Benítez Ormaza; Natalia Andrea Campos Ordóñez; Jaime Alejandro Albán Cuenca; Roberto Adolfo Molina Velastegui

claro propósito de revisar aquellas apreciaciones o investigaciones propuestas por diferentes investigadores relacionadas con el tema de interés, para luego dar la respectiva argumentación a los planteamientos, en función a las necesidades encontradas en la indagación.

\section{Fuentes Documentales.}

El análisis correspondiente a las características que predomina en el tema seleccionado, llevan a incluir diferentes fuentes documentales encargadas de darle el respectivo apoyo y en ese sentido cumplir con la valoración de los hechos a fin de generar nuevos criterios que sirven de referencia a otros procesos investigativos. Para (CASTRO, 2016) las fuentes documentales incorporadas en la investigación documental o bibliográfica, "representa la suma de materiales sistemáticos que son revisados en forma rigurosa y profunda para llegar a un análisis del fenómeno".(p.41). Por lo tanto, se procedió a cumplir con la realización de una lectura previa determinada para encontrar aquellos aspectos estrechamente vinculados con el tema, con el fin de explicar mediante un desarrollo las respectivas apreciaciones generales de importancia.

\section{Técnicas para la Recolección de la Información.}

La conducción de la investigación para ser realizada en función a las particularidades que determinan a los estudios documentales, tiene como fin el desarrollo de un conjunto de acciones encargadas de llevar a la selección de técnicas estrechamente vinculadas con las características del estudio. En tal sentido, (Bolívar, 2015), refiere, que es "una técnica particular para aportar ayuda a los procedimientos de selección de las ideas primarias y secundarias”. (p. 71).

Por ello, se procedió a la utilización del subrayado, resúmenes, fichaje, como parte básica para la revisión y selección de los documentos que presentan el contenido teórico. Es decir, que mediante la aplicación de estas técnicas se pudo llegar a recoger informaciones en cuanto a la revisión bibliográfica de los diversos elementos encargados de orientar el proceso de investigación. Tal como lo expresa, (Bolívar, 2015) "las técnicas documentales proporcionan las herramientas esenciales y determinantes para responder a los objetivos formulados y llegar a resultados efectivos" (p. 58). Es decir, para responder con eficiencia a las necesidades investigativas, se introdujeron como técnica de recolección el método inductivo, que hizo posible llevar a cabo una valoración de los hechos de forma particular para llegar a la explicación desde una visión general. 


\section{Efectos de la obesidad en la reproducción humana femenina}

Vol. 3, núm. 3., (2019)

Yomara Catherine Benítez Ormaza; Natalia Andrea Campos Ordóñez; Jaime Alejandro Albán Cuenca; Roberto Adolfo Molina Velastegui

Asimismo, se emplearon las técnicas de análisis de información para la realización de la investigación que fue ejecutada bajo la dinámica de aplicar diversos elementos encargados de determinar el camino a recorrer por el estudio, según, (Bolívar, 2015)las técnicas de procesamiento de datos en los estudios documentales "son las encargadas de ofrecer al investigador la visión o pasos que debe cumplir durante su ejercicio, cada una de ellas debe estar en correspondencia con el nivel a emplear" (p. 123). Esto indica, que para llevar a cabo el procesamiento de los datos obtenidos una vez aplicado las técnicas seleccionadas, tales como: fichas de resumen, textual, registros descriptivos entre otros, los mismos se deben ajustar al nivel que ha sido seleccionado.

\section{Resultados.}

Un índice de masa corporal (IMC) se considera normal si esta entre 19 - $24.9 \mathrm{~kg} / \mathrm{m} 2$, aunque algunos consideran como normal el límite inferior de $20 \mathrm{~kg} / \mathrm{m} 2$. Ser una persona de bajo peso conduce a la amenorrea hipotalámica y aumenta el riesgo de embarazo si la concepción se produce. El sobrepeso se define como un IMC mayor o igual a $25 \mathrm{~kg} / \mathrm{m} 2$ definiendo la 'pre-obesidad' entre 25.0 - $29.9 \mathrm{~kg} / \mathrm{m} 2$, obesidad moderada (clase I) entre 30.0 - $34.9 \mathrm{~kg} / \mathrm{m} 2$, obesidad severa (clase II) entre 35.0 -39,9 kg / m2 y obesidad muy grave ("morbosa") (clase III) mayor o igual a $40 \mathrm{~kg} /$ $\mathrm{m} 2$.

Por coherencia utilizaremos las definiciones anteriores y se refieren al sobrepeso como un IMC de $25 \mathrm{~kg} / \mathrm{m} 2$ y obesidad con un IMC mayor o igual a $30 \mathrm{~kg} / \mathrm{m} 2$.La causa de la obesidad es un exceso de ingesta energética excesiva, con la mayoría de la población realizando poco ejercicio y comiendo una dieta poco saludable(Lawlor, 2003). Tener padres obesos aumenta el riesgo de obesidad en cinco veces.

La prevalencia de obesidad está aumentando y presentando una carga significativa para la economía y la salud de las personas. El IMC es fácil de medir y una medida reproducible, sin embargo, en términos metabólicos, la distribución de la grasa corporal es más importante que el peso real del cuerpo. La grasa visceral es más metabólicamente activa y un aumento de la circunferencia de la cintura (o relación cintura: cadera) se correlaciona mejor con riesgo metabólico y enfermedad a largo plazo. Desafortunadamente, la circunferencia de la cintura es 


\section{Efectos de la obesidad en la reproducción humana femenina}

Vol. 3, núm. 3., (2019)

Yomara Catherine Benítez Ormaza; Natalia Andrea Campos Ordóñez; Jaime Alejandro Albán Cuenca; Roberto Adolfo Molina Velastegui

difícil de medir (sujeto a mayor error) en individuos obesos, mientras que el IMC es más consistente.

La resistencia a la insulina es un importante correlato del IMC y se percibe como un marcador más preciso del efecto metabólico de la obesidad. También hay importantes variaciones étnicas en la expresión de la resistencia a la insulina. Un IMC de $30 \mathrm{~kg} / \mathrm{m} 2$ generalmente se considera que confieren mayor riesgo en los caucásicos blancos mientras que en los de origen del sur de Asia un IMC inferior $25 \mathrm{~kg} / \mathrm{m} 2$ es suficiente para aumentar el riesgo de defectos metabólicos(Wijeyaratne, 2002).

La resistencia a la insulina se define como una reducción de la glucosa en respuesta a una cantidad dada de insulina y puede ocurrir resistencia secundaria en el receptor de insulina, disminución de la depuración hepática de la insulina y / o aumento en la sensibilidad pancreática. La medida de la resistencia a la insulina es una ciencia imprecisa sin pautas universalmente aceptadas. Dificultades técnicas han dado lugar a una serie de pruebas invasivas incluyendo el método de pinza euglucémica. Esto es considerado como un "patrón oro", pero es complejo y costoso, como es la medición de la concentración rápida de insulina que combinada con glucosa puede proporcionar fórmulas para el cálculo de HOMA y QUICKI de resistencia a la insulina. Así que en la práctica estos se limitan al ámbito de la investigación y no se han establecido en la práctica clínica en la medicina reproductiva. La mayoría de los clínicos recurren a pruebas estándar de tolerancia oral a la glucosa de $75 \mathrm{~g}$ (OGTT).

Efecto de la obesidad en el embarazo (aborto involuntario, salud materna, salud fetal).

Las tasas de aborto espontáneo parecen aumentar con el aumento del peso materno. En los que conciben espontáneamente hay un mayor riesgo de aborto involuntario en los que tienen un sobrepeso moderado (IMC25 - 27,9 kg / m2) (Hamilton-Fairley, 1992).

Esto también se ha demostrado en aquellos que conciben por inseminación o que son receptores de ovocitos donados(Bellver, 2003). 


\section{Efectos de la obesidad en la reproducción humana femenina}

Vol. 3, núm. 3., (2019)

Yomara Catherine Benítez Ormaza; Natalia Andrea Campos Ordóñez; Jaime Alejandro Albán Cuenca; Roberto Adolfo Molina Velastegui

Se ha encontrado una mayor tasa de resistencia a la insulina en mujeres con aborto espontáneo recurrente. Esto introduce la noción que la grasa metabólicamente activa, es decir, la grasa visceral, es la más importante para predecir el resultado reproductivo. Un posible mecanismo es a través del inhibidor del activador del plasminógeno (PAI-1), que es un potente inhibidor defibrinolisis y se eleva en resistencia a la insulina, el PCOS y los que abortan. Hay un número de otros mecanismos posibles, incluyendo efectos de la resistencia a la insulina en el desarrollo folicular, maduración de ovocitos y desarrollo del endometrio.

El embarazo conlleva riesgos significativos para quienes son obesos, con tasas crecientes de anomalías congénitas(tubo neural (OR 3.5), onfalocele (OR 3.3)y defectos cardíacos (OR 2.0), aborto espontáneo, diabetes gestacional, hipertensión y problemas durante el nacimiento (Cedergren, 2004). El riesgo de anomalías congénitas parece real, aunque existen dificultades técnicas en la evaluación del feto por ecografía porque el tejido adiposo atenúa la señal, el embarazo en sí exacerba cualquier resistencia subyacente a la insulina y como resultado, mujeres con El SOP y / o la obesidad tienen un mayor riesgo de diabetes gestacional

La obesidad se asocia con un mayor riesgo para la madre durante el embarazo. Los riesgos incluyen aumento en la incidencia de hipertensión, diabetes gestacional y trastornos tromboembólicos, así como un aumento en la tasa de cesárea, macrosomía, admisión a cuidados intensivos neonatales, defectos de nacimiento, muerte fetal y todas las muertes perinatales aumentan en los infantes de mujeres obesas (Callaway, 2006). En un estudio del Reino Unido con 287.213 embarazos únicos: 176,923 (61.6\%)fueron peso normal (IMC 20 - 24.9) y 31,276(10.9\%) eran obesos (IMC>=30) La proporción de probabilidades del estudio por(Cedergren, Maternal morbid obesity and the risk of adverse pregnancy outcome, 2004) en aquellos que eran obesos por las siguientes condiciones fueron:

Diabetes gestacional: OR 3.6 (3.25 - 3.98)

Preeclampsia: OR 2.14 (1.85 - 2.47)

Inducción del parto: O 1.70 (1.64 - 1.76) 


\section{Efectos de la obesidad en la reproducción humana femenina}

Vol. 3, núm. 3., (2019)

Yomara Catherine Benítez Ormaza; Natalia Andrea Campos Ordóñez; Jaime Alejandro Albán Cuenca; Roberto Adolfo Molina Velastegui

Cesárea: OR $1.83(1.74-1.93)$

Hemorragia posparto: O 1.39 (1.32 - 1.46)

Infección genital: OR $1.3(1.1$ - 1.6)

Infección del tracto urinario: OR 1.4 (1.2 - 1.6)

Infección de la herida: OR $2.2(2.0$ - 2.6)

Macrosomía: OR $2.36(2.23$ - 2.50)

Muerte intrauterina: OR $1.4(1.14$ - 1.71)

Influencia de la obesidad en la fertilidad natural.

El peso corporal tiene un efecto profundo en el inicio de la pubertad en las niñas y la posterior fertilidad natural. Una cuenta detallada está fuera del alcance de este documento, pero hay algunas excelentes críticas recientes, que incluyen valoraciones de las interrelaciones entre hormonas de acción central y productos activos de tejido adiposo. Mientras que la mayor atención se ha dirigido hacia los efectos de la obesidad en la infertilidad anovulatoria, existe evidencia de que el sobrepeso puede influir en la concepción espontánea en mujeres que ovulan.

SOP, resistencia a la insulina, metformina y efecto de la pérdida de peso.

El síndrome de ovario poliquístico (SOP) afecta al 20 - 25\% de mujeres. La prevalencia parece ir en aumento debido a la actual epidemia de obesidad. El aumento de la obesidad abdominal está correlacionado con menor frecuencia menstrual y fertilidad, junto con una mayor resistencia a la insulina. Varios estudios han demostrado que la pérdida de peso en las mujeres con SOP mejoran el perfil endocrino, ciclo menstrual, tasa de ovulación y probabilidad de un embarazo saludable (Norman, 2004). Incluso una pérdida modesta del 5\% al 10\% del peso corporal total consigue una reducción del 30\% de la grasa central, una mejora en la sensibilidad a la insulina y restablecer la ovulación. La modificación del estilo de vida es claramente un componente clave para la mejora de la función reproductiva para el sobrepeso en mujeres anovulatorias con SOP. 


\section{Efectos de la obesidad en la reproducción humana femenina}

Vol. 3, núm. 3., (2019)

Yomara Catherine Benítez Ormaza; Natalia Andrea Campos Ordóñez; Jaime Alejandro Albán Cuenca; Roberto Adolfo Molina Velastegui

Por lo tanto, se debe alentar la pérdida de peso antes de los tratamientos de inducción de la ovulación, como el citrato clomífero o la terapia de gonadotropina, tanto para mejorar la probabilidad de ovulación y mejorar la respuesta de los ovarios.

Un estudio de (Clark, 1995) examinó el efecto de un programa de pérdida de peso y ejercicio para mujeres con un IMC de $30 \mathrm{~kg} / \mathrm{m} 2$ e infertilidad anovulatoria que eran resistentes al clomífero. El estudio comprendió un horario de ejercicio realista y una alimentación adecuada con un programa de más de 6 meses combinado con reforzamiento positivo. Trece de las 18 mujeres inscritas completaron el estudio, reforzando las dificultades que algunas personas tienen en sostener incluso cambios moderados en el estilo de vida. La pérdida de peso tuvo un efecto significativo sobre la función endocrina, la ovulación y la posibilidad de embarazo. El nivel de insulina en ayunas y las concentraciones séricas de testosterona cayeron y doce de las trece mujeres reanudaron la ovulación; once quedaron embarazada. Así, con el apoyo adecuado, los pacientes pueden ovular de forma espontánea sin terapia médica. Una extensión de este estudio, en mujeres con variedad de diagnósticos, demostraron que, en 60 de 67 sujetos, la pérdida de peso dio lugar a la ovulación espontánea con tasas de aborto involuntario inferiores a las previstas y un ahorro significativo en el costo del tratamiento.

La modificación del estilo de vida es claramente un componente clave en la mejora de la función reproductiva en mujeres con sobrepeso, anovulación y SOP. Es probable que, a partir de perder peso, al estar en balance calorífico negativo, proporcionará beneficio temprano. El uso de agentes reductores o sensibilizadores de insulina ha despertado mucho interés en la gestión de SOP, pero incluso la metformina es menos eficaz para mujeres con anovulación y obesidad extrema, aunque quizás se requiera una dosis más alta que la actualmente prescrita. Muchas mujeres obesas que desean concebir ahora están prescritas con metformina, a menudo con pesos corporales mayores de lo que lo haría ser permisible para el tratamiento para inducir la ovulación.

Los obesos deberán afrontar considerables riesgos adicional es durante el embarazo. ¿Es apropiado tratar estas mujeres con metformina a menos que ya hayan comenzado a perder peso o logró su objetivo? Por lo menos los riesgos del embarazo a la madre y al niño deben ser explicados, entendidos, y gestionado activamente antes de embarcarse en tratamiento. 


\section{Efectos de la obesidad en la reproducción humana femenina}

Vol. 3, núm. 3., (2019)

Yomara Catherine Benítez Ormaza; Natalia Andrea Campos Ordóñez; Jaime Alejandro Albán Cuenca; Roberto Adolfo Molina Velastegui

Es lógico suponer que la terapia que logra una caída en las concentraciones séricas de insulina debería mejorar los síntomas del SOP. En la última década muchos se han realizado estudios para evaluar los efectos de la metformina en la reproducción en pacientes con SOP. La mayoría de los estudios iniciales, sin embargo, fueron los estudios observacionales y cualquier estudio aleatorizado incluyeron un pequeño número de participantes.

Ha sido mostrado que la metformina mejora el hiperandrogenismo y anomalías de la secreción de gonadotropina en mujeres con SOP y también puede restaurar la ciclicidad menstrual. La metformina parece ser menos efectiva en aquellos que son significativamente obesos (IMC $35 \mathrm{~kg}$ / m2) y todavía no hay algoritmos acordados para su uso. Además, no hay acuerdo sobre predictores para respuesta o la dosis apropiada, y si la dosis debe ajustarse para el peso corporal u otros factores.

\section{Conclusiones.}

La obesidad influye en la reproducción humana de varias maneras. La obesidad se asocia con subfertilidad y este efecto es notable ya en IMC $25 \mathrm{~kg} / \mathrm{m} 2$. Hasta un 6 - 9\%de la población femenina en edad fértil sufren del síndrome de ovario poliquístico, es decir, infertilidad anovulatoria, entre quienes más de la mitad son obesos. La cirugía se ha vuelto cada vez más común en mujeres de edad fértil, y plantea un problema específico cuando las mujeres quedan embarazadas. Además, la obesidad también ha llegado a la población embarazada de países desarrollados.

El reto para los clínicos dentro del campo obstétrico es triple; Primero se asocia la obesidad con una serie de riesgos obstétricos que pueden tener consecuencias fatales o a largo plazo tanto para la madre como para la descendencia tales como preeclampsia, parto prematuro, muerte intrauterina, y malformaciones congénitas. En segundo lugar, mientras que la cura obvia para la obesidad es la pérdida de peso, esto no es una estrategia adecuada durante el embarazo cuando las demandas nutricionales del feto están en juego. Finalmente, la cesárea no es la respuesta al problema; los resultados adversos neonatales no se ven afectados por el modo de parto, y los 


\section{Efectos de la obesidad en la reproducción humana femenina}

Vol. 3, núm. 3., (2019)

Yomara Catherine Benítez Ormaza; Natalia Andrea Campos Ordóñez; Jaime Alejandro Albán Cuenca; Roberto Adolfo Molina Velastegui

riesgos anestésicos, que son generalmente elevados en las embarazadas, son aún más inminentes en la obesidad de mujeres embarazadas.

\section{Bibliografía.}

Bellver, J. R. (2003). Obesity and the risk of spontaneous abortion after oocyte donation. Fertility and Sterility, 79., 1136-1140.

Bolívar, J. (2015). Investigación Documental. México. Pax.

Callaway, L. (2006). The prevalence and impact of overweight and obesity in an Australian obstetric population. The Medical Journal of Australia, 184., 56-59.

Castro, J. (2016). Técnicas Documentales. México. Limusa.

Catalano, P. (2010). Obesity, insulin resistance, and pregnancy outcome. Reproduction140365371.

Cedergren, M. (2004). Maternal morbid obesity and the risk of adverse pregnancy outcome. Obsterics and Gynecology, Clinical endocrinology, 103., 219-224.

Cedergren, M. (2004). Maternal morbid obesity and the risk of adverse pregnancy outcome. Obsterics and Gynecology, Clinical endocrinology, 103., 203-219.

Clark, A. M. (1995). Weight loss results in significant improvement in pregnancy and ovulation rates in anovulatory obese women. Human reproduction, 2705-2712.

Davila, A. (2015). Concepto de terminos cientificos. Caracas: Oasis.

Hamilton-Fairley. (1992). Obesity and miscarriage. British Journal Obsterics and Gynaecology, 99-128.

Lawlor, D. A. (2003). Geographical variation in cardiovascular disease, risk factors, and their control in older women. Journal of Epidemiol Community, 57., 134-140.

Norman, R. (2004). Improving reproductive performance in overweight/obese women with effective weight management. Human Reproduction Update, 10., 267-280.

Wijeyaratne, C. (2002). Clinical manifestations and insulin resistance (IR) in PCOS among South Asians and Caucasians: is there a difference? Clinical Endocrinology, 57., 343-350.

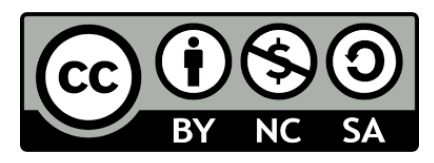

\section{RECONOCIMIENTO-NOCOMERCIAL-COMPARTIRIGUAL}

CC BY-NC-SA

ESTA LICENCIA PERMITE A OTROS ENTREMEZCLAR, AJUSTAR Y CONSTRUIR A PARTIR DE SU OBRA CON FINES NO COMERCIALES, SIEMPRE Y CUANDO LE RECONOZCAN LA AUTORÍA Y SUS NUEVAS CREACIONES ESTÉN BAJO UNA LICENCIA CON LOS MISMOS TÉRMINOS. 